\section{Editorial}

Check for updates

\title{
Impact of Smoking in Survivors from Acute Myocardial Infarction
}

\author{
Hyeon Chang Kim (D, MD, PhD, FAHA
}

Department of Preventive Medicine, Integrative Research Center for Cerebrovascular and Cardiovascular Diseases, Yonsei University College of Medicine, Seoul, Korea

\section{OPEN ACCESS}

Received: Jan 31, 2021

Accepted: Feb 15, 2021

Correspondence to

Hyeon Chang Kim, MD, PhD, FAHA

Department of Preventive Medicine,

Integrative Research Center for

Cerebrovascular and Cardiovascular Diseases,

Yonsei University College of Medicine, 50-1,

Yonsei-ro, Seodaemun-gu, Seoul 03722, Korea.

E-mail: hckim@yuhs.ac

Copyright $($ C 2021. The Korean Society of Cardiology

This is an Open Access article distributed under the terms of the Creative Commons Attribution Non-Commercial License (https:// creativecommons.org/licenses/by-nc/4.0) which permits unrestricted noncommercial use, distribution, and reproduction in any medium, provided the original work is properly cited.

ORCID iDs

Hyeon Chang Kim (D)

https://orcid.org/0000-0001-7867-1240

\section{Funding}

The author received no financial support for the research, authorship, and/or publication of this article.

\section{Conflict of interest}

The author has no financial conflicts of interests.

\section{Data Sharing Statement}

Data sharing is not applicable to this article as no new data were created or analyzed.
- See the article "Effects of Smoking on Long-Term Clinical Outcomes and Lung Cancer in Patients with Acute Myocardial Infarction” in volume 51 on page 336.

Cardiovascular disease and cancer are the leading causes of death in the world. ${ }^{1)}$ Currently in Korea, malignancy is the most common cause of death and heart disease is the second most common cause of death. Because cardiovascular disease and cancer share some risk factors, cancer patients can be considered as a high-risk group of cardiovascular disease, and cardiovascular disease patients also can be a high-risk group for cancer. ${ }^{233}$ Because of the improving survival of cancer patients and the increasing number of cancer survivors, prevention of cardiovascular disease has become an important issue in the management of cancer ${ }^{244} \mathrm{As}$ the survival after major cardiovascular diseases, such as acute myocardial infarction (AMI), is getting longer, there is also a need for evidence for cancer prevention in cardiovascular disease, but there is only limited evidence. ${ }^{5(6)}$ In this issue of Korean Circulation Journal, Lee et al. ${ }^{7}$ provides important data on the effects of smoking on long-term clinical outcomes and lung cancer risk among patients with AMI. Lung cancer and AMI account for the largest proportion of cancer and cardiovascular deaths, respectively. Cigarette smoking is an important modifiable risk factor that contributes greatly to both of the disease. $\left.{ }^{8}\right)$ However, there is little data on the long-term effects of smoking on morbidity and mortality in patients with AMI. In those who survive AMI, smokers are often observed to have lower or similar mortality rates compared to non-smokers. This is presumed to be due to the fact that AMI patients who smoked had fewer other risk factors than AMI patients who did not smoke, but it is difficult to prove with real world data.

Lee and colleagues ${ }^{7}$ used a well-established AMI registry and control the effects of other risk factors using the propensity score matching technique to compare 5-year mortality and morbidity between smoking and non-smoking patients. Before propensity score matching, smokers showed lower risk of all-cause mortality, heart disease mortality, and major adverse cardiovascular events (MACE) rates than non-smokers. However, after the propensity score matching, cigarette smoking was associated with $24 \%$ higher risk of all-cause mortality, $19 \%$ higher risk of heart disease mortality, and 13\% higher risk of MACE. As expected, lung cancer risk was 2 to 3 times higher in smoking patients than in non-smoking patients before and after the propensity score matching." Recently, an Israeli study reported an association between smoking and cancer incidence in AMI survivors and reported that quitting smoking even after AMI could reduce the risk of cancer. ${ }^{9)}$ Both this study and Lee's work are important real world data that demonstrate how important quitting smoking is for the better long-term management of AMI patients. 
The contents of the report are the author's own views and do not necessarily reflect the views of the Korean Circulation Journal.

\section{REFERENCES}

1. Roth GA, Mensah GA, Johnson CO, et al. Global burden of cardiovascular diseases and risk factors, 1990-2019: update from the GBD 2019 study. J Am Coll Cardiol 2020;76:2982-3021.

PUBMED | CROSSREF

2. Lüscher TF. Cardio-oncology: a new specialty moves to centre stage. Eur Heart J2019;40:1743-6. PUBMED | CROSSREF

3. Aboumsallem JP, Moslehi J, de Boer RA. Reverse cardio-oncology: cancer development in patients with cardiovascular disease. J Am Heart Assoc 2020;9:e013754.

PUBMED | CROSSREF

4. Youn JC, Chung WB, Ezekowitz JA, et al. Cardiovascular disease burden in adult patients with cancer: an 11-year nationwide population-based cohort study. Int J Cardiol 2020;317:167-73. PUBMED | CROSSREF

5. Kim Y, Ahn Y, Cho MC, Kim CJ, Kim YJ, Jeong MH. Current status of acute myocardial infarction in Korea. Korean J Intern Med 2019;34:1-10. PUBMED | CROSSREF

6. Kim RB, Kim HS, Kang DR, et al. The trend in incidence and case-fatality of hospitalized acute myocardial infarction patients in Korea, 2007 to 2016. J Korean Med Sci 2019;34:e322. PUBMED | CROSSREF

7. Lee SN, Choi IJ, Lim S, et al. Effects of smoking on long-term clinical outcomes and lung cancer in patients with acute myocardial infarction. Korean Circ J 2021;51:336-48. CROSSREF

8. Koene RJ, Prizment AE, Blaes A, Konety SH. Shared risk factors in cardiovascular disease and cancer. Circulation 2016;133:1104-14. PUBMED | CROSSREF

9. Lotan K, Goldbourt U, Gerber Y. Smoking status and incidence of cancer after myocardial infarction: a follow-up study of over 20 years. Am J Med 2017;130:1084-91.

PUBMED | CROSSREF 